\title{
T-wave Right Slope Provides a New Angle in the Prediction of Drug-Induced Ventricular Arrhythmias
}

\author{
Editorial to: "Electrocardiographic Predictors of Torsadogenic Risk During Dofetilide or \\ Sotalol Initiation: Utility of a Novel T Wave Analysis Program” by Sugrue A. et al.
}

\author{
Jordi Heijman $^{1}$ • Harry J. G. M. Crijns ${ }^{1}$
}

Published online: 5 October 2015

(C) Springer Science+Business Media New York 2015

Cardiac arrhythmias remain a common cause of death and disability [1]. The normal heart beat requires a precisely coordinated interaction between numerous components and dysfunction of any component can promote arrhythmogenesis. For example, the repolarization duration of the cardiac action potential (AP) and its correlate on the body surface, the QTinterval, are determined by a delicate balance of ion currents (Fig. 1, top panel) [2]. If repolarization duration is too short, cells can become excitable too soon, favoring the development of reentrant tachyarrhythmias. On the other hand, if AP duration (APD) becomes too long, secondary depolarizations can develop before full repolarization (termed early afterdepolarizations; EADs), potentially creating large spatial repolarization gradients and triggering Torsades des Pointes (TdP) arrhythmias [2].

The rapid delayed-rectifier $\mathrm{K}^{+}$-current $\left(\mathrm{I}_{\mathrm{Kr}}\right)$ is a major ion current controlling repolarization of the ventricular AP [3]. Loss-of-function mutations in the $\mathrm{KCNH} 2$ gene, encoding the pore-forming subunit of the $\mathrm{I}_{\mathrm{Kr}}$ channel, represent the second-most common form of the congenital long-QT syndrome, whereas gain-of-function mutations have been associated with short-QT syndrome and familial atrial fibrillation [2, 4]. In addition, many drugs have a high affinity for blocking $\mathrm{I}_{\mathrm{Kr}}$, resulting in an acquired form of the long-QT syndrome with a significant risk of ventricular arrhythmias [3, 5]. As such, all novel potential drugs are tested for inhibition of the

Jordi Heijman

jordi.heijman@maastrichtuniversity.nl

1 Department of Cardiology, CARIM School for Cardiovascular Diseases, Faculty of Health, Medicine, and Life Sciences, Maastricht University, PO Box 616, 6200 MD Maastricht, The Netherlands
$\mathrm{I}_{\mathrm{Kr}}$ channel as part of the standard safety pharmacology screening [5].

Nonetheless, class III antiarrhythmic drugs (AADs) such as dofetilide and sotalol, which have $\mathrm{I}_{\mathrm{Kr}}$ as their predominant target, are commonly used in the treatment of cardiac arrhythmias (e.g., for rhythm control of atrial fibrillation) [6]. Because of the risk of proarrhythmic side effects, patients undergoing treatment with class III AADs undergo telemetric screening to assess the risk of TdP. Excessive prolongation of the QTc interval is currently employed as the standard parameter in this process. However, QT-interval prolonging drugs show marked differences in their torsadogenic potency, indicating that QTc-interval prolongation alone is an imperfect measure for the risk of drug-induced $\operatorname{TdP}[5,7,8]$. Substantial research efforts have been directed to identify other ECG parameters that can support risk assessment for both ventricular and atrial arrhythmias [7-10].

In this issue of Cardiovascular Drugs \& Therapy, Sugrue et al. [11] describe an analysis of numerous T-wave parameters that can potentially distinguish patients susceptible to drug-induced TdP from non-susceptible individuals. Based on ECGs of 13 patients who developed TdP during infusion of dofetilide or sotalol and 26 age-and-sex-matched controls, the authors identify the QTc interval in lead V3 as a good, but not perfect, discriminator of drug-induced TdP, correctly classifying $79 \%$ of individuals [11]. Interestingly, T-wave right slope in lead I is identified as a complimentary parameter that improves discrimination of susceptible individuals to $88 \%$. Although both parameters reflect a macroscopic integration of multiple electrophysiological properties, they can conceptually be related to overall APD and its spatial dispersion. Susceptible individuals have larger APD, increasing the risk of EADs, and larger dispersion, together providing a trigger and substrate for ventricular arrhythmias (Fig. 1). Other Twave parameters, notably the Tpeak-Tend interval that has 
Fig. 1 Inhibition of the rapid delayed-rectifier $\mathrm{K}^{+}$-current $\left(\mathrm{I}_{\mathrm{Kr}}\right)$ by class III antiarrhythmic drugs (AADs) including dofetilide and sotalol produces prolongation of the ventricular action potential (AP) duration (APD). Although there are no significant differences in ventricular repolarization between nonsusceptible and susceptible individuals under baseline conditions, the latter group shows a significantly stronger and more heterogeneous APD prolongation after treatment with class III AADs, resulting in an increased likelihood of torsades des pointes (TdP) arrhythmias. The susceptibility can be assessed non-invasively through changes in the ECG morphology, including QT-interval duration and T-wave right-slope, as shown in the work by Sugrue et al. [11]
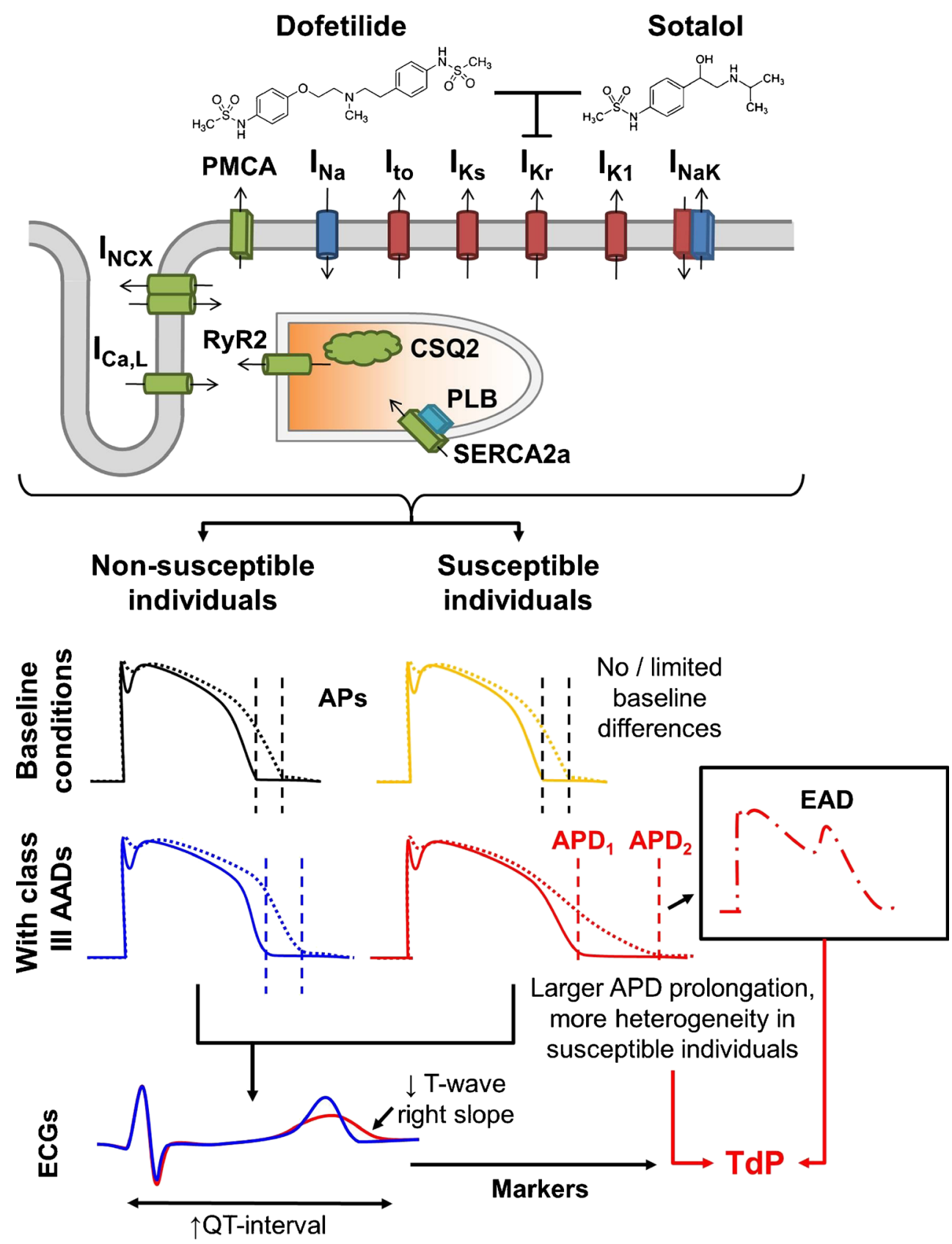

been suggested previously as a marker of TdP risk in several studies [7], might reflect similar electrophysiological concepts, but failed to distinguish cases from controls in the present study [11]. Sugrue et al. suggest that difficulty determining the precise end of the T-wave in the presence of class III AADs might prevent adequate discrimination based on Tpeak-Tend in this small cohort [11]. Although bizarre druginduced T-wave changes $[12,13]$ may at times similarly hinder correct assessment of T-wave right slope, this new parameter - when combined with an accurate QTc measurement might be a more robust parameter that is suitable for (semi)automatic T-wave analyses.
Of note, there were no differences in QTc or T-wave right slope before application of the class III AADs between susceptible and non-susceptible individuals [11], suggesting that the main distinction between groups is their response to dofetilide/sotalol application. There are numerous factors that can influence the repolarization-prolonging effect of drugs, including pharmacokinetic differences affecting the effective concentration within the cardiac muscle, and differences in the electrophysiological substrate on which these drugs act (i.e., the pharmacodynamic response) [8]. In particular, the concept of repolarization reserve has been proposed to capture the redundancy that is present in 
the repolarization of the AP [14]. Because of this redundancy, genetic and/or acquired modulation of other ion channels, which have no or limited effect on the baseline ECG, can modulate the response to class III AADs and the likelihood of TdP [7, 14]. In accordance with this concept, several genetic modifiers of drug-induced TdP have been determined [14]. Whether the response of these ECG parameters to QT-prolonging drugs is influenced by such modifiers remains to be determined.

The cohort employed by Sugrue et al. is small due to the rare occurrence of drug-induced TdP under these conditions with these drugs and confirmation in larger, independent cohorts is clearly needed. Nonetheless, this work advances TdP risk assessment by suggesting a simple and robust parameter that may improve detection of susceptible individuals. In particular, further development of software tools such as those developed in the present study will likely facilitate rapid semi-automated screening of the large amount of ECG data that can be obtained from modern holter recording systems. This may enable longitudinal analyses of the temporal evolution of T-wave parameters during drug administration, which are clearly needed given the absence of baseline differences [11], to identify parameters that could serve as early indicators of impending arrhythmogenesis. Indeed, the work by Sugrue et al. [11] provides preliminary data suggesting that the temporal evolution of QTc interval and $\mathrm{T}$-wave right slope is more pronounced in susceptible patients, but the study is not powered to identify clear cut-offs. Although challenging in practice, such studies could perhaps also be extended with prolonged follow-up periods to further correlate these T-wave parameters with proarrhythmia occurring 'late' during treatment with QTc-prolonging drugs [15].

Taken together, the work by Sugrue et al. [11] has shown that there is still more to be learned from the body-surface ECG. In combination with novel insights about the basic mechanisms of the underlying arrhythmia mechanisms and their modulation by clinical parameters such as sex, age, concomitant heart disease, etc., these data may help to optimize the application of currently available AADs and facilitate the screening of novel drugs, thereby improving the cardiac safety of pharmacological therapies.
Funding None (both authors).

Conflict of Interest None (both authors).

\section{References}

1. Mozaffarian D, Benjamin EJ, Go AS, Arnett DK, Blaha MJ, Cushman M, et al. Heart disease and stroke statistics- 2015 update: a report from the American Heart Association. Circulation. 2015;131(4):e29-322.

2. Bartos DC, Grandi E, Ripplinger CM. Ion channels in the heart. Compr Physiol. 2015;5(3):1423-64.

3. Vandenberg JI, Perry MD, Perrin MJ, Mann SA, Ke Y, Hill AP. hERG $\mathrm{K}^{+}$channels: structure, function, and clinical significance. Physiol Rev. 2012;92(3):1393-478.

4. Napolitano C, Bloise R, Monteforte N, Priori SG. Sudden cardiac death and genetic ion channelopathies: long QT, Brugada, short QT, catecholaminergic polymorphic ventricular tachycardia, and idiopathic ventricular fibrillation. Circulation. 2012;125(16):2027-34.

5. Heijman J, Voigt N, Carlsson LG, Dobrev D. Cardiac safety assays. Curr Opin Pharmacol. 2014;15:16-21.

6. Heijman J, Voigt N, Dobrev D. New directions in antiarrhythmic drug therapy for atrial fibrillation. Futur Cardiol. 2013;9(1):71-88.

7. Sauer AJ, Newton-Cheh C. Clinical and genetic determinants of torsade de pointes risk. Circulation. 2012;125(13):1684-94.

8. Frommeyer G, Eckardt L. Drug-induced proarrhythmia: risk factors and electrophysiological mechanisms. Nat Rev Cardiol. 2015.

9. Couderc JP, Xia X, Peterson DR, McNitt S, Zhao H, Polonsky S, et al. T-wave morphology abnormalities in benign, potent, and arrhythmogenic $\mathrm{I}_{\mathrm{Kr}}$ inhibition. Heart Rhythm. 2011;8(7):1036-43.

10. Lankveld TA, Zeemering S, Crijns HJ, Schotten U. The ECG as a tool to determine atrial fibrillation complexity. Heart. 2014;100(14): 1077-84.

11. Sugrue A, Kremen V, Qiang B, Sheldon SH, DeSimone CV, Sapir $\mathrm{Y}$ et al. Electrocardiographic predictors of torsadogenic risk during dofetilide or sotalol initiation: utility of a novel $t$ wave analysis program. Cardiovasc Drugs Ther. 2015;29:this issue.

12. Houltz B, Darpo B, Edvardsson N, Blomstrom P, Brachmann J, Crijns HJ, et al. Electrocardiographic and clinical predictors of torsades de pointes induced by almokalant infusion in patients with chronic atrial fibrillation or flutter: a prospective study. Pacing Clin Electrophysiol. 1998;21(5):1044-57.

13. Wiesfeld ACP. Risk stratification and management of patients with sustained ventricular tachycardia or ventricular fibrillation (thesis). Groningen: University of Groningen; 1994.

14. Mahida S, Hogarth AJ, Cowan C, Tayebjee MH, Graham LN, Pepper CB. Genetics of congenital and drug-induced long QT syndromes: current evidence and future research perspectives. J Interv Card Electrophysiol. 2013;37(1):9-19.

15. Morganroth J. Early and late proarrhythmia from antiarrhythmic drug therapy. Cardiovasc Drugs Ther. 1992;6(1):11-4. 